\title{
Molecular and Metabolic Imaging in Multiple Sclerosis
}

\author{
Marcello Moccia ${ }^{\mathrm{a}, \mathrm{b}}$ and Olga Ciccarelli, ${ }^{\mathrm{a}, \mathrm{c}}$
}

a. Queen Square MS Centre, University College London, Institute of Neurology, London, United Kingdom

b. MS Clinical Care and Research Centre, Department of Neuroscience, Federico II University, Naples, Italy

c. NIHR University College London Hospitals (UCLH) Biomedical Research Centre (BRC), London, United Kingdom

\section{Corresponding Author:}

${ }^{\mathrm{a}, \mathrm{c}}$ Olga Ciccarelli, PhD, FRCP

NMR Research Unit, Queen Square MS Centre

UCL Institute of Neurology

10-12 Russell Square

WC1B 5EH London

United Kingdom

Phone: +44 2031087415

Fax: +442031087407

o.ciccarelli@ucl.ac.uk (email)

\section{Co-author:}

${ }^{\mathrm{a}, \mathrm{b}}$ Marcello Moccia, MD

NMR Research Unit, Queen Square MS Centre

UCL Institute of Neurology

10-12 Russell Square

WC1B 5EH London

United Kingdom

Phone: +44 2031087449

Fax: +44 2031087407

m.moccia@ucl.ac.uk (email)

\section{DISCLOSURES}

\section{Funding sources}

Prof Ciccarelli : $\quad$ Nil.

Dr Moccia: Nil. 
Conflict of interest

Prof Ciccarelli: $\quad$ is a consultant for Biogen Idec, Genzyme, Novartis, Roche, Teva, and General Electric. She is an associate editor of Neurology ${ }^{\circledR}$.

Dr Moccia: $\quad$ Nil. 


\section{Molecular and Metabolic Imaging in Multiple Sclerosis}

\section{Key words}

Multiple Sclerosis; Magnetic Resonance Imaging; Spectroscopy; Positron Emission Tomography; Molecular; Metabolic.

\section{Synopsis}

Multiple Sclerosis is a multifactorial disease with heterogeneous pathogenetic mechanisms, which deserve to be studied in order to evaluate new possible targets for treatments and improve patient management. MR Spectroscopy and Positron Emission Tomography allow assessing in-vivo the molecular and metabolic mechanisms underlying the pathogenesis of MS. Thus, the present review will focus on the relationship between these imaging techniques and the biological and chemical pathways leading to MS pathology and its clinical features. Future directions of research will be presented.

\section{Key points}

1. Molecular and metabolic imaging techniques assess dynamically and in-vivo the pathogenetic mechanisms that lead to MS pathology and reflect the heterogeneity of pathological abnormalities occurring in MS.

2. ${ }^{1} \mathrm{H}-\mathrm{MR}$ Spectroscopy estimates brain levels of several metabolites, which reflect important biological processes, such as mitochondrial function and/or neuronal integrity (N-Acetyl-Asparate), and glial cell activation and proliferation (Myo-inositol). The concentrations of two neurotransmitters, Gamma-Amino-Butyric Acid, and Glutamate, can also be quantified.

3. ${ }^{23} \mathrm{Na}$ MRI estimates the total concentration of sodium within regions-of-interest in the brain. Increased total sodium concentration is thought to reflect axonal impaired energy metabolism and neurodegeneration. Advanced techniques are needed to distinguish between the intracellular 
and extracellular sodium concentration.

4. Positron Emission Tomography permits the characterisation of the biological processes occurring at the cellular and molecular levels. The following radioligands are often used: ${ }^{18} \mathrm{~F}$ fluoro-2'-deoxyglucose to investigate inflammation, translocator protein tracers to study microglia activation, amyloid tracers to study demyelination, and ${ }^{11} \mathrm{C}$-Flumazenil to investigate neuronal damage.

5. Although technically challenging and expensive, the translation of these techniques to clinical trials and the clinical setting may allow stratification of patients for treatments.

\section{Introduction}

Tags: Multiple Sclerosis; relapsing-remitting; progressive; grey matter; white matter; pathology; molecular and metabolic imaging.

Recent advances in conventional Magnetic Resonance Imaging (MRI) have remarkably improved the diagnosis of multiple sclerosis (MS), which can now be achieved earlier and with greater precision. T2 lesions and brain atrophy estimated with MRI are currently used in clinical trials as outcome measures. However, conventional neuroimaging techniques lack of specificity with regard to different pathophysiological substrates of MS, and are not able to explain the heterogeneous and long-term clinical evolution of this disease. ${ }^{1-4}$

MS usually starts with a relapsing-remitting course (RRMS), characterized by the subacute occurrence of neurological symptoms (namely relapses) that may be followed-up by a clinical improvement. In view of this, the presence of focal areas of inflammatory demyelination in the white matter (WM), known as plaques and responsible for relapses, has been considered the main pathological feature of MS. After 15-25 years from disease onset, the clinical course of MS typically shifts into a progressive course, which is termed secondary progressive MS (SPMS). 
However, a small group of patients (10-15\%) develop a progressive course of the disease since onset, and this type of MS is called primary progressive MS (PPMS).

The main pathological mechanism underling the progressive course of MS is thought to be neurodegeneration. The occurrence of neurodegenerative features are considered to be both a consequence of the inflammatory activity, ${ }^{1,5,6}$ and a primary neurodegenerative component ${ }^{7}$. Neurodegeneration occurs diffusely in the brain and spinal cord of MS patients, and is reflected by changes in structural imaging parameters that can be measured within and outside lesions, namely in the normal appearing WM (NAWM) and grey matter (GM). ${ }^{8}$

The pathogenesis of MS is not fully understood, and it is thought that there is a series of pathobiological events, starting with focal lymphocytic infiltration and microglia activation, and ending with demyelination and neuroaxonal degeneration (Figure 1). In particular, a primary stimulus (either endogenous or exogenous) may be responsible for an inflammatory, demyelinating response, which induces a compensatory response within neurons. This response, which includes the redistribution of sodium channels along the demyelinated axolemma and increased mitochondrial metabolism, might have a transient functional benefit, but may be deleterious in the long-term. Specifically, oxidative stress, mitochondrial injury and ion channel dysfunction have been suggested as possible "maladaptive" changes, leading to neuroaxonal damage (Figure 1). ${ }^{1,9}$ Molecular and metabolic imaging have the unique ability to reflect in-vivo some of the molecular and metabolic pathways involved in the development and progression of neurodegeneration in $\mathrm{MS}^{2,10}$

The present review will describe and discuss clinical studies that used molecular and metabolic imaging techniques to investigate the role of specific molecular, (sub)cellular, and metabolic processes in causing neurodegeneration in MS. The most successful techniques are ${ }^{1} \mathrm{H}-$ MR Spectroscopy (MRS), Sodium imaging, and Positron Emission Tomography (PET); these techniques have been often used to provide insights into the pathogenesis of MS in-vivo and explain the clinical heterogeneity of MS patients. Translation of these techniques to clinical trials and the 
clinical setting may facilitate the development of effective treatments in MS and help to stratify patients for treatments.

\section{MR spectroscopy}

Tags: Metabolic imaging; spectroscopy; proton; sodium; choline; NAA; GABA; Glutamate; MyoInositol.

MR Spectroscopy (MRS) is used to study metabolites in the brain, as long as they are available in relatively high concentrations and present one or more MR visible nuclei (for more details on MRS see the book by Gülin Oz Editor). ${ }^{11}$ The most common nucleus that is used is ${ }^{1} \mathrm{H}$ (proton). In MS, ${ }^{1} \mathrm{H}-\mathrm{MRS}$ is used to provide in-vivo quantification of brain levels of several metabolites. Each metabolite appears at a specific ppm (part per million), and each one reflects specific cellular and biochemical processes; therefore, their concentrations may change in association with pathological abnormalities. The most commonly estimated metabolites are: total Nacetyl-asparate (NAA) (which includes NAA and N-acetylaspartylglutamate), glutamate (Glu), gamma-amino-butyric acid (GABA), creatinine (Cr), choline-containing compounds (Cho) and myo-inositol (Table 1) (Figure 2). These metabolites can be estimated within lesions or in the normal-appearing brain.

We will now review the most recent clinical studies that detected abnormal levels of metabolites in MS patients when compared to healthy controls, by going through the metabolites from the right of the spectrum to the left, and discuss the findings of abnormal levels of metabolites in the brain (lesions, NAWM and GM) and spinal cord.

\section{N-Acetyl-Aspartate}

NAA is synthesized by neural mitochondria and is almost exclusively located within neural cells in the mature brain. ${ }^{2,10,12-14}$ Although its biochemical functions are not completely understood, 
NAA might reflect neuronal mitochondrial metabolism and/or neuronal integrity (Figure 1) ${ }^{10}$.

Since metabolic (mitochondrial) dysfunction is considered to play a role in the neurodegeneration, the in-vivo estimation of NAA levels may provide insights into the mechanisms of damage and recovery in $\mathrm{MS}$.

NAA levels can either be calculated as absolute concentrations or as values normalised to intravoxel $\mathrm{Cr}(\mathrm{NAA} / \mathrm{Cr})$; advantages and disadvantages of both methods have been previously discussed. ${ }^{15}$ In their meta-analytic review including 30 studies evaluating NAA in MS, Caramanos and colleagues found that the directions of change in mean NAA values and mean NAA/Cr ratios were concordant in most studies, suggesting the use of these measures as a practical compromise to the acquisition of a surrogate measure of "cerebral tissue integrity". ${ }^{12,16}$

In acute lesions there is a reduction of NAA levels when compared with the surrounding NAWM,${ }^{17}$ and with the WM of healthy controls, ${ }^{18}$ suggesting neuronal loss and/or metabolic dysfunction; the reduction of NAA may be, at least in part, due to the local oedema, which is associated with acute inflammation. Similarly, in chronic WM lesions (hypointense on T1-weighted images), there is an overall mean decrease in NAA levels of about $20 \% .^{12}$

When the temporal behaviour of NAA is examined over time, MRS studies in the brain and spinal cord have found that reduced NAA levels in acute lesion partially recovers over time, although it does not return to normal. ${ }^{17,19,20}$ Since at the same time, progressive atrophy occurred, it is possible that increased NAA levels indicate the enhanced mitochondrial activity that is necessary to restore and maintain axonal conduction after acute inflammatory demyelination. The resolution of oedema or a change in the relative partial volume of neuronal processes may also play a role in increasing the NAA levels after an acute damage.

In the MS NAWM, there is an overall mean decrease of NAA of about $7 \%$ when compared to healthy $\mathrm{WM} ;{ }^{12}$ greater reductions in NAA were found in the progressive forms of MS. Patients with clinically isolated syndrome (CIS) showed reduced, ${ }^{21,22}$ or normal NAA levels in the NAWM when compared to controls. ${ }^{23,24}$ In a previous study in CIS patients, WM NAA/Cr was significantly 
lower in patients who developed new T2 lesions on MRI and/or relapses during the follow-up than in stable patients, ${ }^{25}$ suggesting that neuroaxonal damage may be already well established in patients at such an early disease stage and thus indicates a more aggressive disease course. Similarly, a relationship between lower NAA levels in several WM regions and higher clinical disability, as assessed by the MSFC, has been described in patients with RRMS. ${ }^{26}$ Interestingly, 18 recently diagnosed, mildly disabled, RRMS patients, all on immunomodulatory medication, showed a $6 \%$ lower WM NAA than controls at study entry, but an increase in NAA over three years, at a rate of $0.1 \mathrm{mM} /$ year; $^{27}$ this increase of NAA levels may indicate a neuronal dysfunction which may improve with treatment.

In the GM, NAA levels were found to be consistently reduced in the thalamus and inconsistently reduced in the cortex of MS patients when compared to healthy controls, ${ }^{12,28-30}$ suggesting neuroaxonal damage and loss. Two different studies conducted in PPMS, RRMS and SPMS patients showed that GM NAA alterations involve the thalamus and the lymbic system, ${ }^{26,29}$ although they might be more diffuse and involve also the prefrontal and motor areas. Lower NAA in the bilateral caudate nucleus was found to correlate with higher clinical disability. ${ }^{26}$

Lower NAA levels were found in the spinal cord of 21 patients with early PPMS when compared to healthy controls, and were associated with higher motor disability, as measured by the EDSS (Expanded Disability Scale Score), ${ }^{31}$ suggesting that neurodegeneration may occur in the spinal cord in the early phase of progressive MS, even in the absence of significant spinal cord atrophy.

Overall, all these studies have supported the use of NAA as marker of axonal loss and/or metabolic dysfunction, and a few clinical trials have used it as a secondary outcome measure to assess the efficacy of experimental treatments in MS. ${ }^{32,33}$ Recommendations and advice on how to introduce the measurement of NAA levels in clinical trials have been previously provided by the MAGNIMS group. $^{15}$ 


\section{Glutamate and Glutamine}

In ${ }^{1} \mathrm{H}-\mathrm{MRS}$, Glu is generally quantified together with Glutamine (Gln), which is both its precursor and catabolite, although Glu makes up the majority of the Glx signal $(\mathrm{Glu}+\mathrm{Gln}=\mathrm{Glx})$. Glu is an important excitatory neurotransmitter, and its in-vivo quantification can be obtained by using specific techniques, allowing unobstructed Glu signal detection.

Increased extracellular Glu concentrations in MS can be determined by enhanced production by inflammatory cells (macrophages and microglial cells), reduced uptake in oligodendrocytes and in astrocytes, and increased receptor expression. Increased Glu levels may contribute to neuroaxonal degeneration, through the mechanism of "excitotoxicity" (Figure 1). ${ }^{10,34}$ An elevation of Glu levels has been found in active WM lesions. ${ }^{35}$

Glu and Gln levels have been found to be increased in the NAWM in different disease subtypes (PPMS, RRMS, and SPMS) when compared with healthy controls. ${ }^{34,36}$ Interestingly, over the course of MS, there is evidence of a progressive reduction in their concentrations within the NAWM, compared to other markers, such as NAA, which, on the opposite, remains substantially stable. Therefore, Glu might increase at an early stage (with suggested toxic effects), and then progressively decline, reflecting progressive neurodegeneration. ${ }^{36}$ A longitudinal study on $343 \mathrm{MS}$ patients (mainly RRMS), showed that higher Glu concentration in the NAWM was associated with higher rate of NAA decline after 2 years, higher rate of brain volume loss after 3 years, and greater disability progression after 4 years, measured with the MS functional composite score. ${ }^{34}$ Therefore, the authors suggested that the Glu/NAA ratio might be a more biologically relevant predictor than either metabolite alone, since when using this ratio the Glu concentrations are adjusted for the amount of the neuronal dysfunction (as measured by NAA). ${ }^{34}$

Glu and Glx concentration have been found to be reduced in the parietal and cingulate GM regions in patients with RRMS when compared to controls. ${ }^{26,37}$ In addition, impairment of the visuospatial memory was significantly associated with lower hippocampal, cingulate and thalamic Glu levels, independently from other measures of GM damage ${ }^{37}$ Similarly, a previous study found 
reduced Glx in the cortical GM in the brains of patients with early PPMS when compared to controls. ${ }^{38}$ Since Glu is more abundant inside cells and in the synaptic terminals than in the extracellular space, a possible interpretation of its reduced concentration is a reduction in the number of synapses and neuroaxonal degeneration, which have been described in post-mortem studies of MS brains. ${ }^{39}$

Reduced Glx levels were also found in the spinal cord of 21 patients with early PPMS when compared to 24 controls, ${ }^{31}$ suggesting that reduced glutamatergic metabolism occur in the early phase of the disease. As mentioned above, it is more challenging to perform MRS and, in particular, to estimate the levels of Glx, in the spinal cord than in the brain.

Blocking Glu receptors seems a promising therapeutical approach to slow down disease progression and, for instance, memantine has been utilized in different clinical trials to improve cognitive function in MS. ${ }^{40}$ In future, the efficacy of medications targeting the glutamatergic metabolism could be monitored by using MRS.

\section{Gamma-Aminobutyric Acid}

GABA is the major inhibitory neurotransmitter in the brain, and can be evaluated with ${ }^{1} \mathrm{H}$ MRS by using spectral editing method that allows its discrimination from more abundant metabolites.

Measurements of GABA in brain lesions and NAWM have not been reported so far.

With regard of the GM, GABA levels have been found to be reduced in the hippocampus and sensorimotor cortex in 30 SPMS patients when compared to 17 healthy controls. ${ }^{41}$ The reduction of GABA in the sensorimotor cortex was associated with a more impaired motor function of the contralateral limbs. ${ }^{41}$ Previous post-mortem studies have detected a loss of GABAergic inhibitory interneurons in the temporal cortex of MS patients with seizures, ${ }^{42}$ and a decrease in presynaptic and postsynaptic components of GABAergic neurotransmission and in the density of inhibitory interneuron processes in MS cortex. ${ }^{43}$ Therefore, GABA has been suggested to be a marker of 
neurodegeneration. Additionally, it is thought that reduced cerebral levels of GABA reflect impaired compensatory mechanisms occurring in the damaged areas of the brain in progressive $\mathrm{MS}^{2,41}$

Agonists of the GABA receptor may be used to manage symptoms associated with MS; for example, baclofen is commonly used to ameliorate spasticity and topiramate is used by some patients with established MS who also have epilepsy or migraines. It has been demonstrated that increasing GABAergic activity may be beneficial for animals with EAE (Experimental Autoimmune Encephalomyelitis) (animal model of MS) and that this effect occurred, at least in part, through a direct effect of the GABAergic agents on the immune system, ${ }^{44}$ suggesting that further studies are needed to establish whether GABAergic agents may have an effect on underlying MS disease activity and whether MRS can be used to monitor it.

\section{Creatine}

Cr reflects energy metabolism. A meta-analysis has reported no significant overall change in the $\mathrm{Cr}$ in MS lesions and GM when compared to WM and GM of healthy controls, and a mediumeffect-sized overall increase in the Cr of NAWM relative to WM. In a recent study, Cr has been found to be increased within the corticospinal tract, which is characterized by a high metabolic rate, of RRMS and PPMS patients when compared to controls. ${ }^{45}$ Lower $\mathrm{Cr}$ levels within the corticospinal tract were associated with higher motor disability in 13 PPMS and 14 RRMS patients, suggesting that an impaired energy metabolism can be responsible for clinical features of MS. ${ }^{18,45}$

\section{Choline-containing compounds}

Cho is a marker of cell membrane, and its increase reflects elevated cellular turnover. It is often reported to be raised in the NAWM of MS patients when compared to controls. ${ }^{27}$ Narayana and colleagues described the elevation of Cho before the appearance of a Gadolinium-enhancing lesion, suggesting that Cho might be a marker of inflammation preceding demonstrable blood-brain 
barrier breakdown. ${ }^{17}$

When Cho is measured along the whole cortico-spinal tract, it was higher in 13 RRMS when compared to 16 healthy controls, but similar between RRMS and PPMS patients. In addition, in the PPMS group, lower Cho levels were associated with worse walking abilities and greater disability, as measured by the EDSS, suggesting that abnormal membrane turnover from demyelination and remyelination, which is reflected by lower Cho levels, might be responsible for disability accrual. ${ }^{45}$ However, these correlations were not confirmed by the study of Kirov and colleagues carried out in 18 RRMS patients with short disease duration and minimal disease activity; additionally, this work did not detect significant changes in Cho levels in the GM between patients and controls, suggesting that the injury may be focal, subtle or hetereogeneous between cortex and deep GM. ${ }^{27}$ Similarly, Donadieu and colleagues did not find any difference in GM Cho levels between 19 RRMS patients and 19 healthy controls, possibly because of their voxelwise between-group analysis, which might have made it difficult to detect the random distribution of Cho. ${ }^{26}$

\section{Myo-inositol}

Myo-inositol is a glial cell marker, and its increase reflects glial cell (or astrocytic) activation and proliferation (Figure 1). ${ }^{10,35,46}$

Different disease subtypes (e.g. PPMS and RRMS) show increased myo-inositol levels in the acute and chronic WM lesions of the brain and the spinal cord when compared to healthy controls. ${ }^{18,26,31,35,47}$ In a study carried out in 14 RRMS patients, higher myo-inositol levels within acute lesions of the spinal cord were associated with greater disability, as measured by the EDSS. ${ }^{18}$

Myo-inositol levels were found to be higher in the NAWM of 19 RRMS when compared to 19 healthy control, especially in the periventricular regions within bilateral temporal and frontal lobes, in the left thalamus and superior temporal gyrus. ${ }^{26}$ A significantly higher myo-inositol concentration was found in the corticospinal tract of 13 RRMS patients when compared to 16 healthy controls, and higher myo-inositol levels were associated with greater walking difficulties. 
However, in the same study, no difference was found between PPMS and healthy controls, suggesting a more widespread glial activation in the corticospinal tract of patients with RRMS. ${ }^{45} \mathrm{~A}$ study carried out in patients with clinically isolated syndrome (CIS) has reported elevated myoinositol levels in the NAWM when compared to control subjects, ${ }^{23}$ which did not correlate with T2 lesion load, suggesting that myo-inositol may reflect a process of pathogenic importance, independently of WM lesions. However, other studies in CIS patients did not confirm increased myo-inositol in NAWM when compared to controls. ${ }^{21,24}$

Higher myo-inositol levels were found in the cortical GM and in the thalamus of 19 RRMS patients when compared to controls, ${ }^{26}$ suggesting that astrocytic activation and proliferation involve both cortical and deep GM regions in MS.

Finally, myo-inositol levels have been found to be increased in the lesional upper cervical cord of MS patients, when compared to controls and to patients with neuromyelitis optica (NMO), ${ }^{47}$ with potentially important diagnostic implications. Reduced myo-inositol values might reflect the extent of the damage of astrocytes, which are highly damaged in NMO, but not in MS, where they are typically activated and increased in number (i.e. gliosis).

\section{Limitations}

From a technical point of view, there are several limitations of MRS imaging, ${ }^{11}$ which may hamper their use in the clinical setting and in clinical trials. ${ }^{15}$ These include: 1) long acquisition times, more significantly for chemical-shift imaging (CSI) than for single-voxels MRS; 2) lower spatial resolution compared to $\mathrm{MRI},{ }^{46}$ with minimal spatial information provided by single voxel MRS acquisition; 3) difficulties in differentiating between the intracellular and the extracellular pool of different metabolites and between GM and WM compartments within volumes of interest; 4) use of different scanners and acquisition protocols which can be responsible for incomplete agreement on metabolite concentrations; 5) spectra are influenced by variations in B0 and B1 magnetic fields, and there are pathology-related changes in the $\mathrm{T} 1$ and $\mathrm{T} 2$ relaxation times of the 
spectral metabolites that need to be considered when investigating patients. Furthermore, spinal cord MRS presents additional technical limitations, such as the small size of the cord, the susceptibility artefacts due to tissue-bone interfaces, and the motion artefacts arising from respiratory, arterial and cardiac activities, and systolic-related CSF and spinal cord pulsations.

\section{Sodium imaging}

In the central nervous system (CNS), sodium concentration is maintained at a markedly lower concentration $(12 \mathrm{mM} / \mathrm{L})$ in the larger intracellular compartment (tissue fraction of $80 \%$ ), when compared to the higher concentration $(140 \mathrm{mM} / \mathrm{L})$ in the smaller extracellular compartment (tissue fraction of 20\%), as a result of complex energy-dependent mechanisms (e.g. Na/K pump) (Figure

1). ${ }^{1}$ Increased concentration of intracellular sodium is caused by impaired mitochondrial metabolism and is considered to play a role in the cascade of event that leads to neuronal death. Additionally, in acute inflammatory demyelinated lesions, there is extracellular oedema, with increased fluid in the interstitial compartments. Increased extracellular sodium in MS may also occur because of the expansion of the extracellular space secondary to neuroaxonal loss.

Previous sodium MRI studies have estimated the total sodium concentration in the brain, without distinguishing between its intracellular and extracellular component. ${ }^{48,49}$ These studies have reported higher total sodium concentrations in lesions, NAWM and cortical GM in MS patients when compared to controls, with especially high concentrations detected in patients with higher clinical disability and progressive MS. ${ }^{49}$ These findings suggest that raised total sodium concentration may reflect neuroaxonal damage, which is responsible for progression and increased disability. However, also patients with early RRMS show abnormally high total sodium concentrations, although limited to a certain number of brain regions (brainstem, bilateral cerebellum, left temporal pole) and the GM. ${ }^{50}$

More recently, the use of ultra-high field MRI has allowed the discrimination of intracellular and extracellular sodium concentrations. Petracca and colleagues have performed a triple-quantum 
filtered (TQF) ${ }^{23} \mathrm{Na}$ MRI with a MR pulse sequence and applied a single quantum and TQF imaging at $7 \mathrm{~T}$ to quantify intracellular sodium concentration and intracellular sodium volume fraction, an indirect measure of the extracellular sodium. Both total and intracellular sodium concentrations were increased in the NAWM and in the GM of 19 RRMS, compared to 17 controls, whereas extracellular sodium concentrations were reduced; furthermore, reduced levels of extracellular sodium were associated with disability, as measured by the EDSS. ${ }^{48}$ These findings suggest that sodium accumulation is not only a consequence of demyelination and neuroaxonal loss, but also a marker of metabolic dysfunction. Therefore, measuring the intracellular component might provide the unique opportunity to identify metabolically dysfunctional brain areas, which might be targeted by new drugs restoring their function and, so, preventing irreversible structural alterations.

\section{Positron Emission Tomography}

Tags: Molecular imaging; Positron Emission Tomography; radioligand; tracer; FDG; amyloid; myelin; acetate; adenosine; choline; Flumazenil; GABA; TSPO.

PET is a quantitative imaging technique, which investigates cellular and molecular processes in vivo. It requires the reconstruction of an image of the tissue of interest after the distribution of an exogenously administrated positron-emitting molecule, ideally binding a selective target. ${ }^{46,51}$

As MS is a complex and multifactorial disease, there are different possible radioligands, which can be used in PET imaging with relatively high selectivity. Examples of the targets of the PET radioligands used in MS are shown in Figure 1.

We now discussed the results of the studies using PET tracers by grouping them according to the underlying pathological processes that they reflect: inflammation, demyelination, neuronal damage and astrocyte activation (Table 2). 


\section{Inflammatory markers}

The acute, inflammatory activity in MS is characterised by breakdown of the blood-brain barrier, inflammatory cell infiltrates, microglial activation, and production of immune soluble mediators and harmful inflammatory enzymes. In the short-term, inflammation is responsible for the development of acute demyelinating lesions, with consequent clinical relapses. ${ }^{9,10}$ In the longterm, demyelinated axons undergo neurodegeneration. Additionally, microglial cell activation is thought to have a short-term positive effect on tissue repair, but a long-term contribution to neuronal cell death. ${ }^{13,46,52}$ These inflammatory processes may be associated with increased PET signal detected in MS patients when compared to healthy controls.

TSPO PET. The Translocator Protein (TSPO, formerly known as the peripheral benzodiazepine receptor or PBR) is an $18 \mathrm{kDa}$ macromolecular complex, which is expressed at low levels in the outer mitochondrial membrane and is strongly up-regulated in activated microglial cells. Hence, TSPO ligands can reflect in-vivo the presence of activated microglial cells. ${ }^{13,46,52,53}$ TSPO studies using ${ }^{11} \mathrm{C}-\mathrm{PK} 11195$ (a 3-isoquinolinecarboxamide antagonist with nanomolar affinity for TSPO) have reported increased uptake of this radiotracer in active MS lesions and in the NAWM than healthy controls, ${ }^{54,55}$ suggesting that this radiotracer might detect the widespread microglial cell activation in the active inflammatory rim of chronically active plaques and in the NAWM. Increased ${ }^{11} \mathrm{C}-\mathrm{PK} 11195$ uptake has been found also in the cortical GM of 10 RRMS and 8 SPMS patients when compared to 8 healthy controls. ${ }^{54}$ Higher TSPO binding levels were associated with disability scores, particularly if located in clinically meaningful anatomic regions. ${ }^{55}$

A few PET studies were carried out in small groups of MS patients using the second generation of TSPO tracers, such as ${ }^{11} \mathrm{C}$-vinpocetine, ${ }^{11} \mathrm{C}-\mathrm{PBR} 28,{ }^{18} \mathrm{~F}$-FEDAA1106, ${ }^{18} \mathrm{~F}$-PBR111 and ${ }^{18} \mathrm{~F}$-DPA714. An example of a PET scan showing the ${ }^{11} \mathrm{C}$-PBR28 uptake in MS is given in

Figure 3. These novel radioligands are expected to overcome the relatively low brain uptake and non-specific binding of ${ }^{11} \mathrm{C}-\mathrm{PK} 1119$ with subsequent higher signal-to-noise ratios. ${ }^{10,46,51,53,56}$ However, the TSPO polymorphisms, which explains differences in binding affinity of the PET 
radioligand between subjects, needs to be considered when using the second generation TSPO ligands. ${ }^{13,57}$ Increased uptake of ${ }^{18}$ F-PBR111 was detected in WM lesions and NAWM of 11 MS patients, when compared with 11 healthy controls. ${ }^{56}$ Reduced uptake of ${ }^{18}$ F-PBR111 in the hippocampus of 13 RRMS patients, when compared to 22 healthy controls, was associated with higher scores at the Beck Depression Inventory, suggesting the involvement of this area in mood symptoms of MS. ${ }^{58}$ In an exploratory analysis comparing 4 RRMS patients with 4 healthy controls, ${ }^{11} \mathrm{C}-\mathrm{PBR} 28$ presented a good test-retest reproducibility (less than $9 \%$ ), and so might be suitable for future longitudinal assessments. ${ }^{59}$

${ }^{18}$ F-FDG PET. Brain 2'-[ $\left[{ }^{18} \mathrm{~F}\right]$ fluoro-2'-deoxyglucose $\left({ }^{18} \mathrm{~F}\right.$-FDG) PET measures the cerebral metabolic rate of glucose utilization and, hence, has been employed as a marker of inflammation. Higher metabolic rates are found in regions with ongoing inflammation due to an avid uptake from activated inflammatory cells. ${ }^{13,51}$ Specifically, ${ }^{18}$ F-FDG PET has been utilised in MS to classify WM lesions as either acute (hyper-metabolism) or chronic (hypo-metabolism) based on local glucose metabolism. ${ }^{60}$

However, ${ }^{18}$ F-FDG uptake is not specific for inflammatory cells and is limited by the high background glucose uptake within the brain, which reduces its ability to detect small active lesions. ${ }^{13,51}$ It has been suggested that ${ }^{18}$ F-FDG uptake reflects glucose transport and metabolism by neurons and astrocytes, and, so, their integrity and functional state. ${ }^{10,60}$ In agreement with this hypothesis, a pilot ${ }^{18}$ F-FDG PET study of the spinal cord in 8 MS patients reported a lower uptake of this radioligand when compared to 8 healthy controls. Furthermore, a decreased ${ }^{18}$ F-FDG binding was associated with worse autonomic and walking symptoms, ${ }^{61}$ suggesting that an impaired metabolic activity in the spinal cord may be responsible for neurological disability.

Purine PET. Adenosine is a ubiquitous purine that binds to a group of G-protein-coupled receptors (A1R, A2AR, A2BR, and A3R). A2A receptors are upregulated at sites of inflammation and tissue damage, thereby attenuating inflammation. ${ }^{51,62}{ }^{11} \mathrm{C}-\mathrm{TMSX}$ radioligand binds to adenosine A2A receptors. Rissanen and colleagues have carried out a PET study using this tracer in 10 SPMS 
patients and 10 age- and sex-matched healthy controls. ${ }^{62}$ They found increased distribution volumes of ${ }^{11} \mathrm{C}$-TMSX in the NAWM of SPMS when compared with controls. Furthermore, the increased A2AR binding was associated with increased tissue loss and reduced fiber integrity, as measured by diffusion tensor imaging, and higher disability, as measured by the EDSS. ${ }^{62}$

It is worth noting that this technique might be particularly valuable in measuring the impact of treatments modulating the adenosine pathways, such as caffeine-related compounds. ${ }^{51,63-65}$

Purinergic pathways can be studied also by targeting the P2X7 receptor. Different tracers have been developed (e.g. $\left.{ }^{11} \mathrm{C}-J \mathrm{NJ}-54173717,{ }^{11} \mathrm{C}-\mathrm{A}-740003,{ }^{11} \mathrm{C}-\mathrm{GSK}-1482160\right),{ }^{57,66,67}$ and some of them have been tested on animal models of MS. ${ }^{57,66}$ These tracers might measure the activation of microglia and the associated release of inflammatory cytokines, and are expected to be tested soon in-vivo. ${ }^{68}$

\section{Myelin markers}

Demyelination is a hallmark of MS and can occur in both GM and WM. The inflammatory process causes the development of acute demyelinating lesions, which is associated with axonal transection. ${ }^{69}$ Demyelination can be followed by remyelination, or, in the long-term, neurodegeneration. ${ }^{9}$

Amyloid PET. PET images with amyloid tracers, such as a carbon-labelled version of the Pittsburgh Compound B $\left({ }^{11} \mathrm{C}-\mathrm{PIB}\right)$ and ${ }^{18}$ F-Florbetaben, show normal uptake in healthy WM, thereby making the amyloid tracers potentially useful for studying MS. ${ }^{51,70,71}$

In acute WM lesions, ${ }^{11} \mathrm{C}$-PIB and ${ }^{18} \mathrm{~F}$-Florbetaben uptake was decreased when compared to NAWM, and a decreasing gradient in binding levels was found when going from the NAWM to the lesions, ${ }^{72,73}$ reflecting a more extensive myelin loss in acute lesions than in the NAWM. Both positive and negative variations in the binding of these PET ligands were observed in the chronic phase of the WM lesions, reflecting the net result of dynamic processes of myelin loss and remyelination. Additionally, in patients with RRMS, a higher ${ }^{11} \mathrm{C}-\mathrm{PIB}$ uptake within the WM 
lesions was associated with a more benign clinical evolution. ${ }^{72}$ Progressive forms of MS showed a more pronounced reduction of the uptake in the damaged WM, in comparison to the RRMS type. ${ }^{73}$ These studies suggest that in future amyloid-PET may be used to monitor MS progression and may be used to provide outcome measures in clinical trials designed to measure the effects of remyelinating agents. $^{46,71}$

${ }^{11} \mathrm{C}$-BMB $\left({ }^{11} \mathrm{C}\right.$-1,4-bis(p-aminostyryl)-2-methoxybenzene) and its derivatives (i.e. ${ }^{11} \mathrm{C}$-CIC, along with $\left.{ }^{11} \mathrm{C}-\mathrm{MeDAS}\right)$, which have showed promising results in animal models of MS, with concentration-dependent binding to WM tracts, will be investigated in humans. ${ }^{13,52}$

\section{Neuronal markers}

Neurodegenerative alterations occur even in the early stages of MS. Chronic modifications include activation of glial cells, infiltration of macrophages and, ultimately, neuronal functional alterations and death. ${ }^{1}$ In view of this, a variety of PET radioligands has been utilized to measure different components of these chronic neurodegenerative changes.

${ }^{11}$ C-Flumazenil PET. ${ }^{11} \mathrm{C}$-Flumazenil is an antagonist of the central benzodiazepine receptor, a component of the ubiquitous GABA-A receptor complex present in the neuronal synapses in the GM. Thus, ${ }^{11} \mathrm{C}$-Flumazenil PET is a specific marker of neuronal integrity. ${ }^{2,13,74}$

In a pilot study, Freeman and colleagues found a reduced ${ }^{11} \mathrm{C}$-Flumazenil cortical binding in 9 RRMS and 9 SPMS patients, compared to 8 healthy controls. Interestingly, this difference was already seen in RRMS, who did not show a significant degree of GM atrophy. In addition, reduced GABA-A receptor binding was associated with impairment of information processing speed, ${ }^{74}$ further suggesting the clinical relevance of this quantitative measure. These findings suggest that synaptic/dendritic damage may occur prior to a quantifiable grey matter volume loss, and contribute to clinical impairment. In future, neuroprotective and reparative treatment trials that aim to stop or slow down the progression of MS may consider developing the ${ }^{11} \mathrm{C}$-Flumazenil cortical binding as outcome measure for clinical trials or to select the patients who show early neurodegenerative 
changes in the GM.

\section{Markers of astrocyte activation}

Glial (or astrocytic) cell activation and proliferation is known to occur since the early stage of MS, as discussed above in the myo-inositol section. Astrocytes are thought to play an important role in the pathogenesis of MS. ${ }^{75}$

${ }^{11}$ C-Acetate PET. In MS lesions, activated glial cells overexpress metabolic enzymes, such as the monocarboxylate transporter, ${ }^{76}$ which absorbs acetate into astrocytes. The acetate is then converted into fatty acids. Takata and colleagues carried out an ${ }^{11} \mathrm{C}$-Acetate PET study in 6 RRMS patients and 6 healthy controls. An increased uptake of this radioligand was observed in both the WM and the GM of MS patients when compared to controls, but was more pronounced in the WM, particularly in the regions of axonal damage, as detected by diffusion-tensor imaging MRI. ${ }^{77}$ Therefore, the detection of acetate has been suggested as a marker of astrocytic-associated damage in MS, although a development of a clinically feasible protocol for acetate PET and future investigations are warranted.

Choline PET. A preliminary study by Virta and colleagues did not find any difference in the cortical uptake of the PET tracer ${ }^{11} \mathrm{C}-\mathrm{MP} 4 \mathrm{~A}$, that reflects the acetylcholinesterase (AChE) activity, between 10 SPMS patients and 10 healthy controls. ${ }^{78}$ Nevertheless, ${ }^{11} \mathrm{C}$-Choline and ${ }^{18} \mathrm{~F}$-Choline PET can evaluate the cholinergic metabolic profile of specific areas, such as large demyelinated lesions. $^{79,80}$

\section{Limitations}

Possible limitations of PET imaging include: 1) the injection of radioactive tracers, although generally at low dosages; 2) PET costs which are still high for its application in the clinical practice; 3) lack of standardized procedures (there is a need for data modelling techniques based on methods not dependent on reference plasma input or manual delineation of reference tissue region); ${ }^{54} 4$ ) 
relatively low resolution of PET with subsequent difficulties in evaluating small plaques; ${ }^{77}$ 5) static image acquisition in most protocols with subsequent difficulties in tracking the tracer over time.

\section{Conclusions}

Tags: Molecular and metabolic imaging; MR Spectroscopy; Positron Emission Tomography; Multiple Sclerosis; treatment; clinical trial; drug development; pathology; future.

Despite the high costs of PET and the technical challenges of PET and MRS studies, these techniques have provided insights into the pathogenesis of MS and helped to understand the clinical heterogeneity of MS patients. The next step is to translate these techniques from the research laboratories to clinical trials and the clinical setting, in order to identify groups of patients who are more likely to respond to a medication and to monitor the treatment response. For example, reparative clinical trials testing the efficacy of remyelinating agents may in future benefit from inclusion of myelin PET into their trial design, perhaps to select patients who have the greatest remyelination potential.

\section{References}

1. Friese MA, Schattling B, Fugger L. Mechanisms of neurodegeneration and axonal dysfunction in multiple sclerosis. Nat Rev Neurol. 2014;10(4):225-238.

2. Bodini B, Louapre C, Stankoff B. Advanced imaging tools to investigate multiple sclerosis pathology. Press Medicale. 2015;44(4):e159-e167.

3. Sormani MP, Arnold DL, De Stefano N. Treatment effect on brain atrophy correlates with treatment effect on disability in multiple sclerosis. Ann Neurol. 2014;75(1):43-49.

4. Popescu V, Agosta F, Hulst HE, et al. Brain atrophy and lesion load predict long-term 
disability in multiple sclerosis. J Neurol Neurosurg Psychiatry. 2013;84(10):1082-1091.

5. Inglese M, Oesingmann N, Casaccia $\mathrm{P}$, et al. Progressive multiple sclerosis and gray matter pathology: An MRI perspective. Mt Sinai J Med. 2011;78(2):258-267.

6. Scalfari A, Neuhaus A, Daumer M, et al. Early relapses, onset of progression, and late outcome in multiple sclerosis. JAMA Neurol. 2013;70(2):214-222.

7. Stys P, Zamponi G, van Minnen J, et al. Will the real multiple sclerosis please stand up? Nat Rev Neurosci. 2012;13(7):507-514.

8. Koini M, Filippi M, Rocca M, et al. Correlates of Executive Functions in Multiple Sclerosis Based on Structural and Functional MR Imaging: Insights from a Multicenter Study. Radiology. 2016;280(3):869-879.

9. Dendrou C, Fugger L, Friese M. Immunopathology of multiple sclerosis. Nat Rev Immunol. 2015;15(9):545-558.

10. Ciccarelli O, Barkhof F, Bodini B, et al. Pathogenesis of multiple sclerosis: Insights from molecular and metabolic imaging. Lancet Neurol. 2014;13(8):807-822.

11. Gülin Ö. Magnetic Resonance Spectroscopy of Degenerative Brain Diseases. Contempora. Springer; 2016.

12. Caramanos Z, Narayanan S, Arnold DL. 1H-MRS quantification of tNA and $\mathrm{tCr}$ in patients with multiple sclerosis: A meta-analytic review. Brain. 2005;128(11):2483-2506.

13. Matthews PM, Comley R. Advances in the molecular imaging of multiple sclerosis. Expert Rev Clin Immunol. 2009;5(6):765-777.

14. van Horssen J, Witte M, Ciccarelli O. The role of mitochondria in axonal degeneration and tissue repair in MS. Mult Scler J. 2012;18(8):1058-1067.

15. De Stefano N, Filippi M, Miller D, et al. Guidelines for using proton MR spectroscopy in multicenter clinical MS studies. Neurology. 2007;69(20):1942-1952.

16. Roosendaal SD, Barkhof F. Imaging Phenotypes in Multiple Sclerosis. Neuroimaging Clin N Am. 2015;25(1):83-96. 
17. Narayana PA, Doyle TJ, Lai D, et al S. Serial proton magnetic resonance spectroscopic imaging, contrast-enhanced magnetic resonance imaging, and quantitative lesion volumetry in multiple sclerosis. Ann Neurol. 1998;43(1):56-71.

18. Ciccarelli O, Wheeler-Kingshott CA, McLean MA, et al. Spinal cord spectroscopy and diffusion-based tractography to assess acute disability in multiple sclerosis. Brain. 2007;130(8):2220-2231.

19. Ciccarelli O, Altmann DR, McLean MA, et al. Spinal cord repair in MS: Does mitochondrial metabolism play a role? Neurology. 2010;74(9):721-727.

20. De Stefano N, Matthews P, Antel J, et al. Chemical pathology of acute demyelinating lesions and its correlation with disability. Ann Neurol. 1995;38(6):901-909.

21. Wattjes MP, Harzheim M, Lutterbey GG, et al. High field MR imaging and 1H-MR spectroscopy in clinically isolated syndromes suggestive of multiple sclerosis: Correlation between metabolic alterations and diagnostic MR imaging criteria. J Neurol. 2008;255(1):56-63.

22. Rocca MA, Mezzapesa DM, Falini A, et al. Evidence for axonal pathology and adaptive cortical reorganization in patients at presentation with clinically isolated syndromes suggestive of multiple sclerosis. Neuroimage. 2003;18(4):847-855.

23. Fernando KTM, McLean MA, Chard DT, et al. Elevated white matter myo-inositol in clinically isolated syndromes suggestive of multiple sclerosis. Brain. 2004;127(6):13611369.

24. Brex PA, Gomez-Anson B, Parker GJM, et al. Proton MR spectroscopy in clinically isolated syndromes suggestive of multiple sclerosis. J Neurol Sci. 1999;166(1):16-22.

25. Sbardella E, Tomassini V, Stromillo M, et al. Pronounced focal and diffuse brain damage predicts short-term disease evolution in patients with clinically isolated syndrome suggestive of multiple sclerosis. Mult Scler J. 2011;17(12):1432-1440.

26. Donadieu M, Le Fur Y, Lecocq A, et al. Metabolic voxel-based analysis of the complete 
human brain using fast 3D-MRSI: Proof of concept in multiple sclerosis. J Magn Reson Imaging. 2016;44(2):411-419.

27. Kirov II, Tal A, Babb JS, et al. Serial proton MR spectroscopy of gray and white matter in relapsing-remitting MS. Neurology. 2013;80(1):39-46.

28. Chard DT, Griffin CM, McLean MA, et al. Brain metabolite changes in cortical grey and normal-appearing white matter in clinically early relapsing-remitting multiple sclerosis. Brain. 2002;125(Pt 10):2342-2352.

29. Geurts JJG, Reuling IEW, Vrenken H, et al. MR spectroscopic evidence for thalamic and hippocampal, but not cortical, damage in multiple sclerosis. Magn Reson Med. 2006;55(3):478-483.

30. Tiberio M, Chard DT, Altmann DR, et al. Metabolite changes in early relapsing-remitting multiple sclerosis: A two year follow-up study. J Neurol. 2006;253(2):224-230.

31. Abdel-Aziz K, Schneider T, Solanky BS, et al. Evidence for early neurodegeneration in the cervical cord of patients with primary progressive multiple sclerosis. Brain. 2015;138(Pt 6):1568-1582.

32. Filippi M, Rocca MA, Pagani E, et al. Placebo-controlled trial of oral laquinimod in multiple sclerosis: MRI evidence of an effect on brain tissue damage. J Neurol Neurosurg Psychiatry. 2014;85(8):852-859.

33. Sajja BR, Narayana PA, Wolinsky JS, et al. Longitudinal magnetic resonance spectroscopic imaging of primary progressive multiple sclerosis patients treated with glatiramer acetate: multicenter study. Mult Scler. 2008;14(1):73-80.

34. Azevedo CJ, Kornak J, Chu P, et al. In vivo evidence of glutamate toxicity in multiple sclerosis. Ann Neurol. 2014;76(2):269-278.

35. Srinivasan R, Sailasuta N, Hurd R, et al. Evidence of elevated glutamate in multiple sclerosis using magnetic resonance spectroscopy at 3 T. Brain. 2005;128(5):1016-1025.

36. MacMillan EL, Tam R, Zhao Y, et al. Progressive multiple sclerosis exhibits decreasing 
glutamate and glutamine over two years. Mult Scler J. 2016;22(1):112-116.

37. Muhlert N, Atzori M, De Vita E, et al. Memory in multiple sclerosis is linked to glutamate concentration in grey matter regions. J Neurol Neurosurg Psychiatry. 2014;85:834-840.

38. Sastre-Garriga J, Ingle GT, Chard DT, et al. Metabolite changes in normal-appearing gray and white matter are linked with disability in early primary progressive multiple sclerosis. Arch Neurol. 2005;62(4):569-573.

39. Wegner C, Esiri MM, Chance SA, et al. Neocortical neuronal, synaptic, and glial loss in multiple sclerosis. Neurology. 2006;67(6):960-967.

40. Lovera J, Ramos A, Devier D, et al. Polyphenon E, non-futile at neuroprotection in multiple sclerosis but unpredictably hepatotoxic: Phase I single group and phase II randomized placebo-controlled studies. J Neurol Sci. 2015;358(1-2):46-52.

41. Cawley N, Solanky BS, Muhlert N, et al. Reduced gamma-aminobutyric acid concentration is associated with physical disability in progressive multiple sclerosis. Brain. 2015;138(9):2584-2595.

42. Nicholas R, Magliozzi R, Campbell G, et al. Temporal lobe cortical pathology and inhibitory GABA interneuron cell loss are associated with seizures in multiple sclerosis. Mult Scler. 2016;22(1):25-35.

43. Dutta R, McDonough J, Yin X, et al. Mitochondrial dysfunction as a cause of axonal degeneration in multiple sclerosis patients. Ann Neurol. 2006;59(3):478-489.

44. Bhat R, Axtell R, Mitra A, et al. Inhibitory role for GABA in autoimmune inflammation. Proc Natl Acad Sci U S A. 2010;107(6):2580-2585.

45. Tur C, Wheeler-Kingshott CAM, Altmann DR, et al. Spatial variability and changes of metabolite concentrations in the cortico-spinal tract in multiple sclerosis using coronal CSI. Hum Brain Mapp. 2014;35(3):993-1003.

46. Matthews PM, Datta G. Positron-emission tomography molecular imaging of glia and myelin in drug discovery for multiple sclerosis. Expert Opin Drug Discov. 2015;10(5):557-570. 
47. Ciccarelli O, Thomas DL, De Vita E, et al. Low Myo-inositol indicating astrocytic damage in a case series of neuromyelitis optica. Ann Neurol. 2013;74(2):301-305.

48. Petracca M, Vancea RO, Fleysher L, et al. Brain intra- and extracellular sodium concentration in multiple sclerosis: A 7 T MRI study. Brain. 2016;139(3):795-806.

49. Paling D, Solanky BS, Riemer F, et al. Sodium accumulation is associated with disability and a progressive course in multiple sclerosis. Brain. 2013;136:2305-2317.

50. Zaaraoui W, Konstandin S, Bertrand A, et al. Distribution of Brain Sodium Accumulation Correlates with Disability in Multiple Sclerosis: A Cross-sectional 23Na MR Imaging Study. Radiology. 2012;264(3):859-867.

51. De Paula Faria D, Copray S, Buchpiguel C, et al. PET imaging in multiple sclerosis. J Neuroimmune Pharmacol. 2014;9(4):468-482.

52. Niccolini F, Su P, Politis M. PET in multiple sclerosis. Clin Nucl Med. 2015;40(1):e46-e52.

53. Janssen B, Vugts DJ, Funke U, et al. Imaging of Neuroinflammation in Alzheimer's Disease, Multiple Sclerosis and Stroke: Recent Developments in Positron Emission Tomography. Biochim Biophys Acta. 2015;1862(3):425-441.

54. Rissanen E, Tuisku J, Rokka J, et al. In Vivo Detection of Diffuse Inflammation in Secondary Progressive Multiple Sclerosis Using PET Imaging and the Radioligand C-11PK11195. J Nucl Med. 2014;55(6):939-944.

55. Politis M, Giannetti P, Su P, et al. Increased PK11195 PET binding in the cortex of patients with MS correlates with disability. Neurology. 2012;79(6):523-530.

56. Colasanti A, Guo Q, Muhlert N, et al. In Vivo Assessment of Brain White Matter Inflammation in Multiple Sclerosis with (18)F-PBR111 PET. J Nucl Med. 2014;55(7):11121118.

57. Janssen B, Vugts DJ, Funke U, et al. Synthesis and initial preclinical evaluation of the P2X7 receptor antagonist [11C]A-740003 as a novel tracer of neuroinflammation. J Label Compd Radiopharm. 2014;57(8):509-516. 
58. Colasanti A, Guo Q, Giannetti P, et al. Hippocampal Neuroinflammation, Functional Connectivity, and Depressive Symptoms in Multiple Sclerosis. Biol Psychiatry. 2015;80(1):62-72.

59. Park E, Gallezot JD, Delgadillo A, et al. 11C-PBR28 imaging in multiple sclerosis patients and healthy controls: test-retest reproducibility and focal visualization of active white matter areas. Eur J Nucl Med Mol Imaging. 2015;42(7):1081-1092.

60. Schiepers C, Van Hecke P, Vandenberghe R, et al. Positron emission tomography, magnetic resonance imaging and proton NMR spectroscopy of white matter in multiple sclerosis. Mult Scler. 1997;3(1):8-17.

61. Kindred JH, Koo PJ, Rudroff T. Glucose uptake of the spinal cord in patients with multiple sclerosis detected by (1)(8)F-fluorodeoxyglucose PET/CT after walking. Spinal Cord. 2014;52(Suppl 3):S11-S13.

62. Rissanen E, Virta JR, Paavilainen T, et al. Adenosine A2A receptors in secondary progressive multiple sclerosis: a [(11)C]TMSX brain PET study. J Cereb Blood Flow Metab. 2013;33(9):1394-1401.

63. Hedström AK, Mowry EM, Gianfrancesco MA, et al. High consumption of coffee is associated with decreased multiple sclerosis risk; results from two independent studies. J Neurol Neurosurg Psychiatry. 2016;

64. Liu Y, Zou H, Zhao P, et al. Activation of the adenosine A2A receptor attenuates experimental autoimmune encephalomyelitis and is associated with increased intracellular calcium levels. Neuroscience. 2016;330:150-161.

65. Wang T, Xi NN, Chen Y, et al. Chronic caffeine treatment protects against experimental autoimmune encephalomyelitis in mice: Therapeutic window and receptor subtype mechanism. Neuropharmacology. 2014;86:203-211.

66. Ory D, Celen S, Gijsbers R, et al. Preclinical evaluation of a P2X7 receptor selective radiotracer: PET studies in a rat model with local overexpression of the human P2X7 
receptor and in non-human primates. J Nucl Med. 2016;

67. Gao M, Wang M, Green MA, et al. Synthesis of [11C]GSK1482160 as a new PET agent for targeting P2X7 receptor. Bioorganic Med Chem Lett. 2015;25(9):1965-1970.

68. Hagens M, van Berckel B, Barkhof F. Novel MRI and PET markers of neuroinflammation in multiple sclerosis. Curr Opin Neurol. 2016;29:229-236.

69. Trapp B, Peterson J, Ransohoff R, et al. Axonal Transection in the Lesions of Multiple Sclerosis. N Engl J Med. 1998;338(5):278-285.

70. Stankoff B, Freeman L, Aigrot MS, et al. Imaging central nervous system myelin by positron emission tomography in multiple sclerosis using [methyl-11C]-2-(4-methylaminophenyl)- 6hydroxybenzothiazole. Ann Neurol. 2011;69(4):673-680.

71. Mallik S, Samson RS, Wheeler-Kingshott CAM, et al. Imaging outcomes for trials of remyelination in multiple sclerosis. J Neurol Neurosurg Psychiatry. 2014;85(12):1396-1404.

72. Bodini B, Veronese M, García-Lorenzo D, et al. Dynamic imaging of individual remyelination profiles in multiple sclerosis. Ann Neurol. 2016;

73. Matías-Guiu JA, Cabrera-Martín MN, Matías-Guiu J, et al. Amyloid PET imaging in multiple sclerosis: an 18F-florbetaben study. BMC Neurol. 2015;15(1):243.

74. Freeman L, Garcia-Lorenzo D, Bottin L, et al. The neuronal component of gray matter damage in multiple sclerosis: A [(11) C]flumazenil positron emission tomography study. Ann Neurol. 2015;78(4):554-567.

75. Ludwin SK, Rao VT, Moore CS, et al. Astrocytes in multiple sclerosis. Mult Scler J. 2016;22(9):1114-1124.

76. Nijland PG, Michailidou I, Witte ME, et al. Cellular distribution of glucose and monocarboxylate transporters in human brain white matter and multiple sclerosis lesions. Glia. 2014;62(7):1125-1141.

77. Takata K, Kato H, Shimosegawa E, et al. 11C-Acetate PET imaging in patients with multiple sclerosis. PLoS One. 2014;9(11):e111598. 
78. Virta JR, Laatu S, Parkkola R, et al. Cerebral acetylcholinesterase activity is not decreased in MS patients with cognitive impairment. Mult Scler. 2011;17(8):931-938.

79. Padma MV, Adineh M, Pugar K, et al. Functional imaging of a large demyelinating lesion. J Clin Neurosci. 2005;12(2):176-178.

80. Bolcaen J, Acou M, Mertens K, et al. Structural and metabolic features of two different variants of multiple sclerosis: A PET/MRI study. J Neuroimaging. 2013;23(3):431-436. 
Figure 1. Mechanisms of neurodegeneration and the molecular and metabolic imaging targets studied in MS.

Inflammatory cells (e.g. lymphocytes, monocytes, microglia) produce reactive oxygen species and reactive nitrogen species, which contribute to mitochondrial injury. This leads to metabolic stress, energy deficiency and progressive loss of neuro-axonal function. Activation and proliferation of astrocytes occur to restore neuro-axonal function, although reactive astroglial cells also have harmful consequences on axonal survival. After acute demyelination, there is a redistribution of ion channels (e.g. $\mathrm{Na}^{+}$channels), that, along with accumulation of Glutamate (the main excitatory neurotransmitter of the CNS), promotes ionic imbalance, with increased intracellular concentration of calcium and consequent neuronal apoptosis. The metabolites most commonly studied in MS are highlighted in the red boxes, and the targets of PET radioligands are shown in the blue boxes.

MS: Multiple Sclerosis; CNS: Central Nervous System; MRS: Magnetic Resonance Spectroscopy; PET: Positron Emission Tomography.

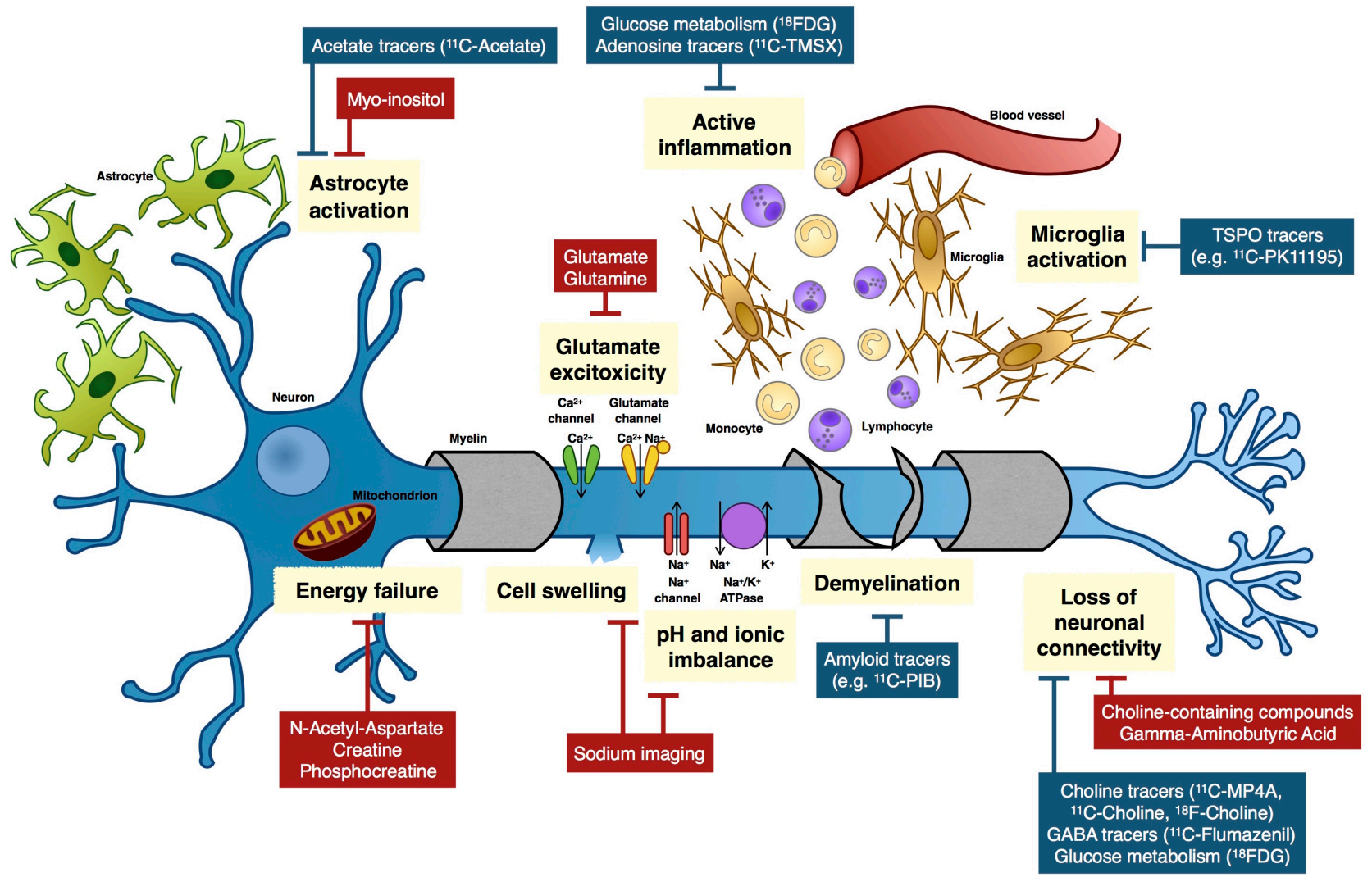


Figure 2. Examples of the spectra on MRS in the corticospinal tract.

Anatomy of the corticospinal tract (A), with the corresponding chemical shift imaging grid on a single image (B); spectrum from the voxel located above the left cerebral peduncle in a healthy control (C) (from Tur et al 2014, permission to reproduce requested).

MRS: Magnetic Resonance Spectroscopy.
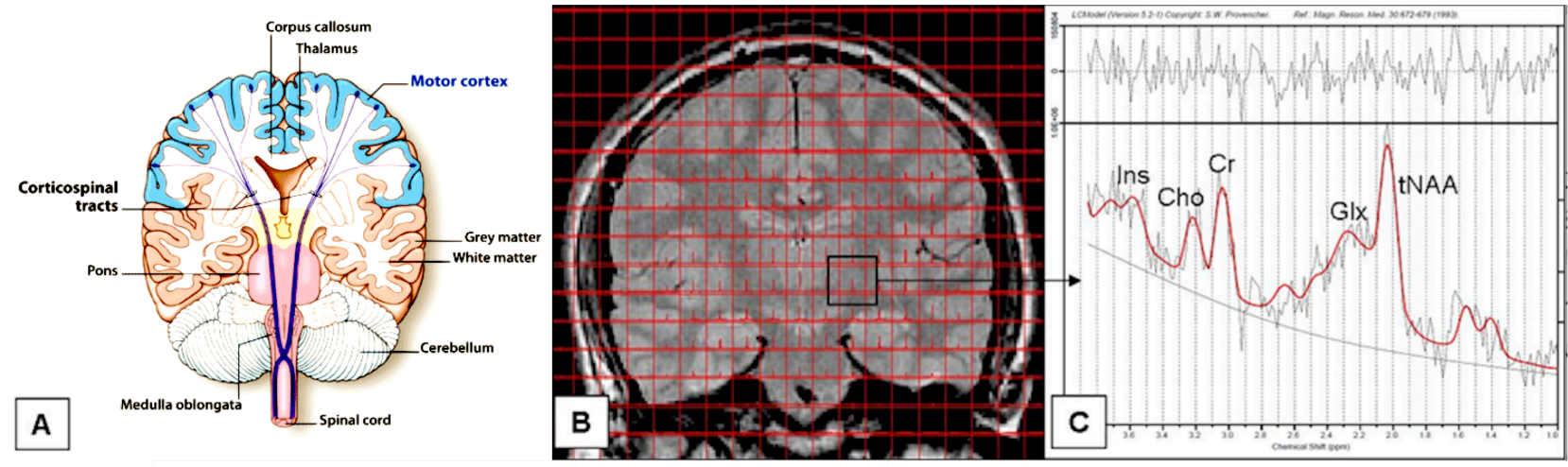


\section{Figure 3. Example of TSPO PET scans in MS.}

T1 axial section from a 45 year old female patient with aggressive disease course (disease duration of 14 years) (A), and a 42 year old female patient with benign disease course with EDSS of 1.0 (B). The corresponding T1 overlaid with voxel-wise TSPO PET image of ${ }^{11} \mathrm{C}-\mathrm{PBR} 28$ distribution volume ratio (DVR) for patient A (C) and patient B (D). Bright yellow indicates high DVR and red is lower DVR. Overlaid on the images from (C) and (D) in (E) and (F), respectively, are the normal appearing white matter (in green) and T2 white matter lesion (in blue) masks (Courtesy of Dr Gourab Datta and Prof Paul Matthews, Imperial College London, UK).

TSPO PET: Translocator Protein Positron Emission Tomography
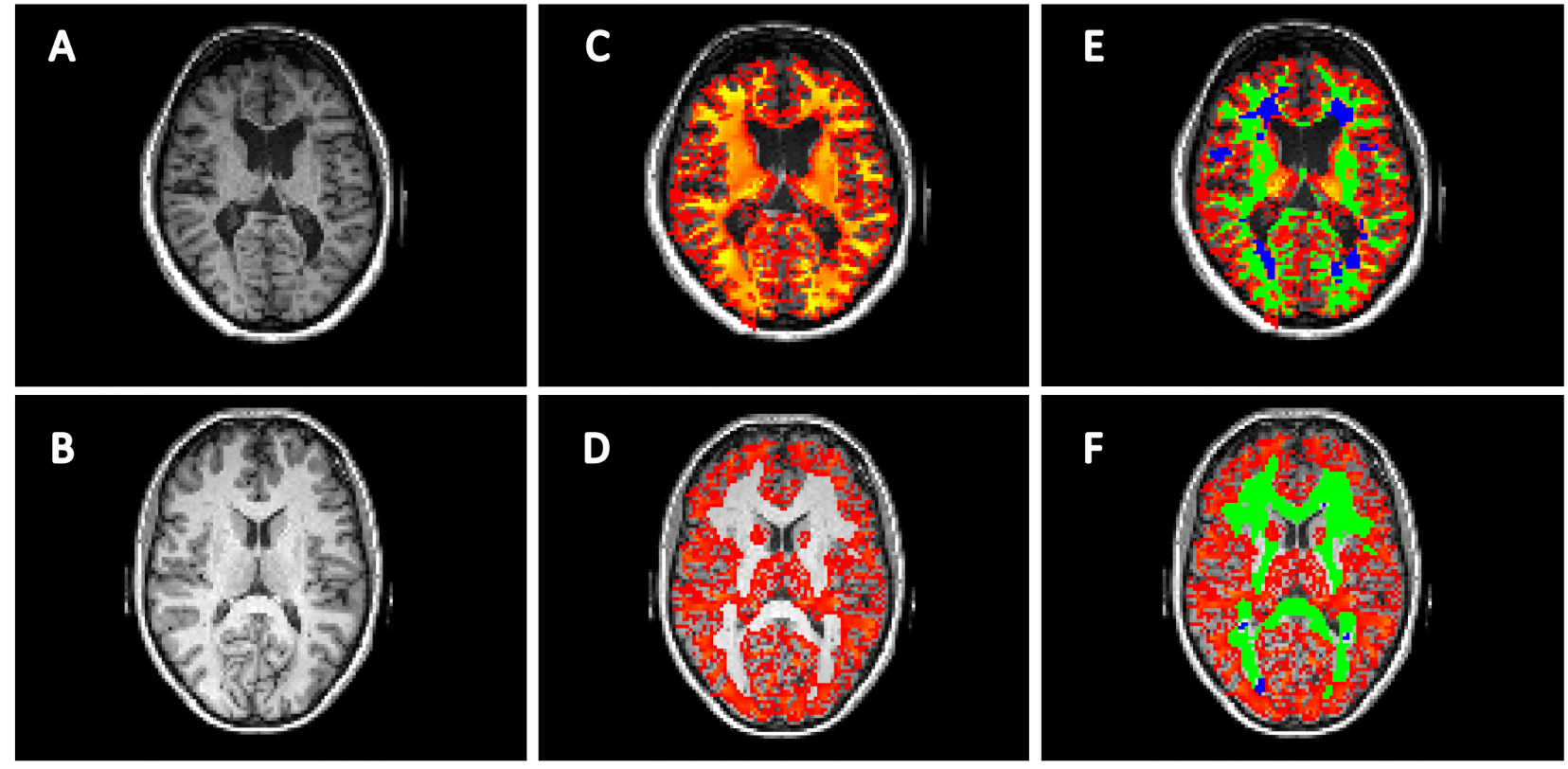